\title{
ANATOMIC AND PHYSIOLOGIC CORRECTION OF THE RESTRICTED POSTERIOR MITRAL LEAFLET MOTION IN CHRONIC ISCHEMIC MITRAL REGURGITATION
}

\author{
Mircea Dobre, MD, ${ }^{\mathrm{a}}$ Bansi Koul, MD, PhD, ${ }^{\mathrm{a}}$ and Anders Rojer, MD, ${ }^{\mathrm{b}}$ Lund, Sweden
}

Mitral valve replacement in chronic ischemic mitral regurgitation (MR) is associated with a 30-day mortality as high as $15 \% .{ }^{1}$ Mitral ring annuloplasty alone in patients with chronic ischemic MR and more than $2+{ }^{*}$ MR has been shown to be attended with a significant residual MR in $10 \%$ to $28 \%$ of patients. $^{2,3}$ Direct suture between the anterior mitral leaflet (AML) and posterior mitral leaflet (PML) at the posteromedial commissure (A3 to P3) ${ }^{\dagger}$ commissuroplasty, has also been tried in chronic ischemic MR with varying success. ${ }^{2}$

In this brief communication we are discussing a new method to repair the restricted PML in patients with chronic ischemic MR, which not only corrects the restricted PML motion but also offers a better coaptation between the mitral leaflets and does not compromise the effective orifice area of the mitral orifice.

Clinical summaries

PATIENT 1. Patient 1 was a 58-year-old woman with a body surface area (BSA) of $1.5 \mathrm{~m}^{2}$, Canadian Cardiovascular Society (CCS) class III angina pectoris, and New York Heart Association (NYHA) functional class III heart failure. Preoperative transthoracic echocardiography demonstrated a restricted PML motion corresponding to P2 and P3 of the PML. Other preoperative and follow-up echocardiographic findings are summarized for both patients in Table I. On March 12, 1997, she underwent coronary artery bypass grafting $(\mathrm{CABG})$ and mitral valve replacement, as per details outlined separately. She is now free from angina and in NYHA functional class I. The postoperative transesophageal echocardiogram shows a good opening of the mitral orifice (Fig 1) and a normal coaptation of the mitral leaflets (Fig 2).

PATIENT 2. Patient 2 was a 57-year-old man with a BSA of $2.04 \mathrm{~m}^{2}$ and CCS class III angina in NYHA functional class III. Preoperative transesophageal echocardiography showed (Fig 3) restricted PML motion corresponding to P2 and P3

From the Departments of Thoracic and Cardiovascular Surgery, ${ }^{\mathrm{a}}$ and Cardiology, ${ }^{\mathrm{b}}$ University Hospital Lund, Sweden.

Received for publication Nov 22, 1999; accepted for publication Feb $16,2000$.

Address for reprints: Bansi Koul, Department of Thoracic and Cardiovascular Surgery, University Hospital in Lund, Lund, Sweden.

J Thorac Cardiovasc Surg 2000;120:409-11

*For explanation, see legend to Table I.

$\dagger$ For explanation, see legend to Fig 2.

Copyright (C) 2000 by The American Association for Thoracic Surgery

$0022-5223 / 2000 \$ 12.00+0 \quad \mathbf{1 2 / 5 4 / 1 0 6 5 2 1}$

doi: $10.1067 / \mathrm{mtc} .2000 .106521$

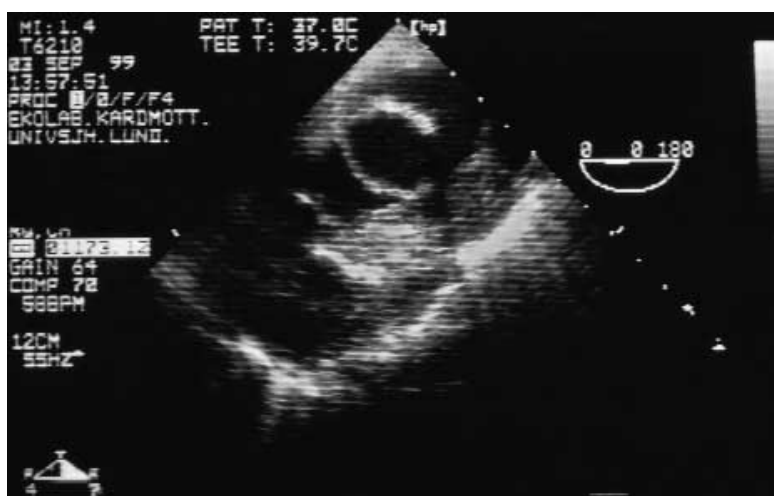

Fig 1. Postoperative transesophageal echocardiogram of patient 1 . Good opening of the mitral orifice with some calcification on the autologous pericardial patch used for enlargement.

with false prolapse of the AML corresponding to A2 and A3. He underwent surgery on February 16, 1999, with 2 vein grafts and mitral repair. He is also now free from angina and in NYHA class I. The postoperative transesophageal echocardiogram shows adequate coaptation of the mitral leaflets (Fig 4).

Mitral valve repair. The mitral valve was repaired with the use of cardiopulmonary bypass, moderate hypothermia, and blood cardioplegia after conventional cannulation of the superior and inferior venae cavae and ascending aorta. The left atrium was approached through the sulcus of Sondergaard, and the mitral valve was exposed and examined. Mitral valve repair was begun by detaching the medial half of $\mathrm{P} 2$, the entirety of $\mathrm{P} 3$, and the posteromedial commissural leaflet from the corresponding parts of the mitral anulus. Before patch enlargement of the PML, ring annuloplasty mattress sutures were placed in the posterior mitral anulus. An approximately $50 \times 14-\mathrm{mm}$ patch of pericardium (autologous pericardium in patient 1 and bovine pericardium [Supple Periguard Axima Stille, Stockholm, Sweden] in patient 2) was cut to a sickle shape and sewn circumferentially in the PML defect with continuous 6-0 Prolene sutures (Ethicon, Inc, Somerville, NJ) anteriorly toward the detached PML and 5-0 Prolene sutures posteriorly toward the posterior mitral anulus (Fig 5). A Cosgrove-Edwards mitral annuloplasty ring (Baxter Healthcare Corporation, Irvine, Calif; 28 $\mathrm{mm}$ in patient 1 and $30 \mathrm{~mm}$ in patient 2) was used to reinforce the posterior mitral valve anulus on the basis of the measurement of the AML.

Discussion. After a transmural posteromedial myocardial infarction, the dilatation of the left ventricle and the mitral 


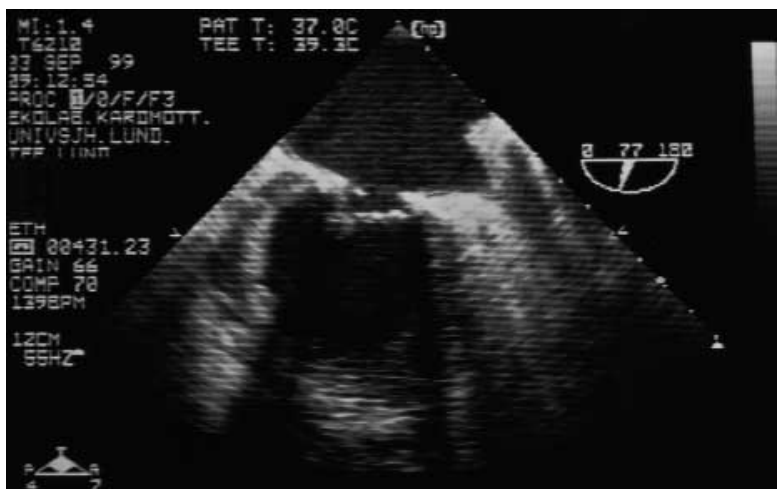

Fig 2. Postoperative transesophageal echocardiogram of patient 1 , showing a good coapation of the mitral leaflets (near P3).

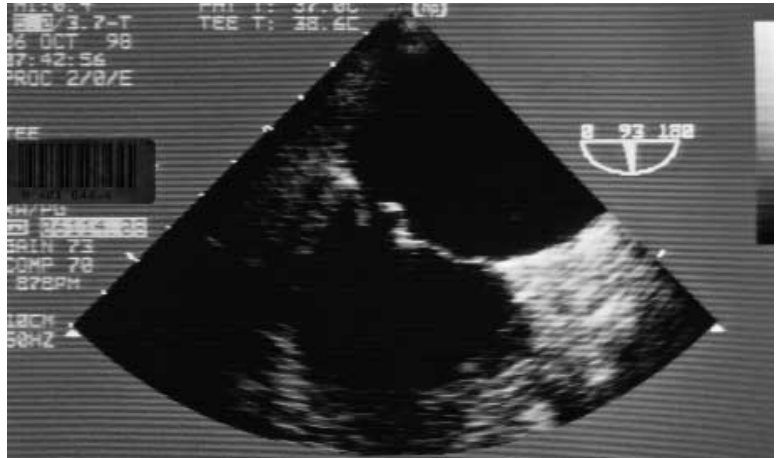

Fig 3. Preoperative transesophageal echocardiogram of patient 2, showing restricted PML motion and false prolapse of the AML.

Table I. Echocardiography data

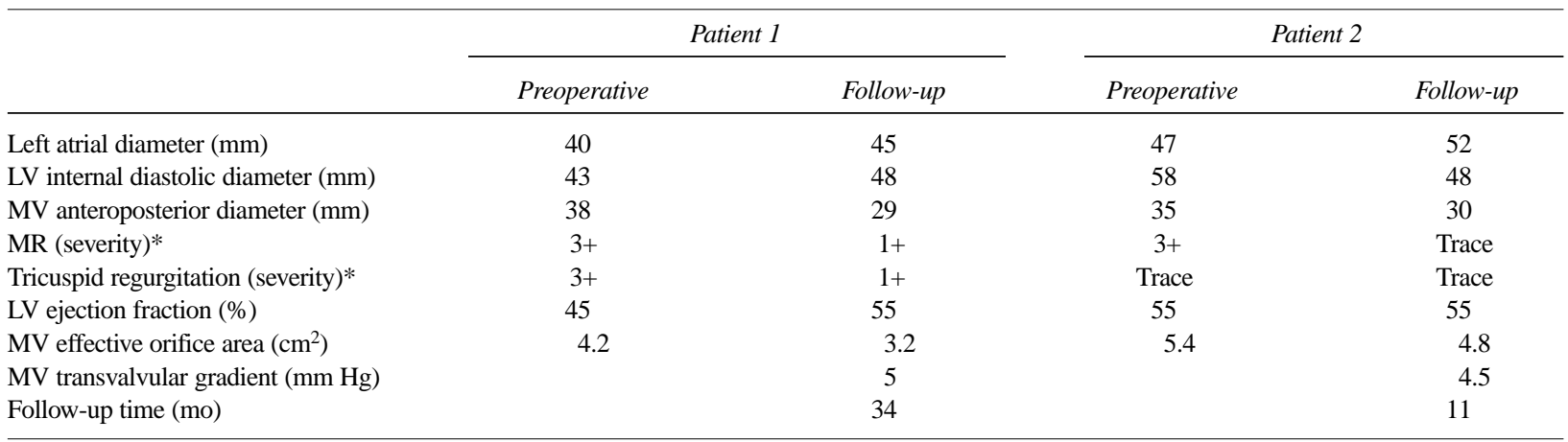

$L V$, Left ventricular; $M V$, mitral valve; $M R$, mitral regurgitation.

$* 1+$, Mild; $2+$, moderate; $3+$, moderate to severe; $4+$, severe.

anulus is asymmetric. The infarcted posterior left ventricular wall bulges outward in systole and drags the posteromedial papillary muscle away with it. The tethering forces on the chordae tendineae emerging from the affected posteromedial papillary muscle are increased, resulting in restricted PML motion corresponding to the medial half of $\mathrm{P} 2$, the entirety of $\mathrm{P} 3$, and the posteromedial commissure. This results in a vicious coaptation between the AML and the PML and thereby MR. ${ }^{4-6}$ Studies done at the Institute for Bioengineering and Biosciences, Georgia Institute of Technology, Atlanta, Georgia, showed that during systole, normal mitral valve leaflets coapt over a height of $8 \pm 1.5 \mathrm{~mm}$. Furthermore, a progressive symmetric mitral annular dilatation by as much as $80 \%$ is well tolerated and does not lead to any MR (coaptation reserve). However, in the presence of an asymmetric tethering of a papillary muscle to the myocardium (reduced coaptation reserve), an annular dilatation of already less than $40 \%$ is sufficient to cause MR. This may explain why in patients with chronic ischemic MR and more than 2+ MR, mitral ring annuloplasty alone, which corrects the underlying defect only partially, is attended with a significant residual MR in up to $28 \%$ of cases. ${ }^{3}$

Another surgical technique used presently in chronic ischemic MR is "downsizing" of the mitral valve with mitral annuloplasty rings that are one to two sizes smaller than would have normally been used. This procedure transforms the mitral valve into a "monocuspid valve," with the AML as the sole functioning leaflet. This procedure ought to produce restriction of the entire PML and, in time, lead to a progressive increase in the tethering forces on the chordae and papillary muscles. Moreover, this procedure may make the mitral valve stenotic in relation to the patient BSA, affecting adversely both early and medium-term results. Sufficiently adequate follow-up of this procedure is not available as yet.

Patch enlargement of the restricted PML ought to restore both the normal extent of mitral leaflet coaptation and PML motion. We chose to use a pericardial patch with a width of $14 \mathrm{~mm}$ so that, at best, the patch provides a new coaptation surface of about $10 \mathrm{~mm}$, taking into account a loss of about 2-mm patch width on either side in the suture line itself. The 


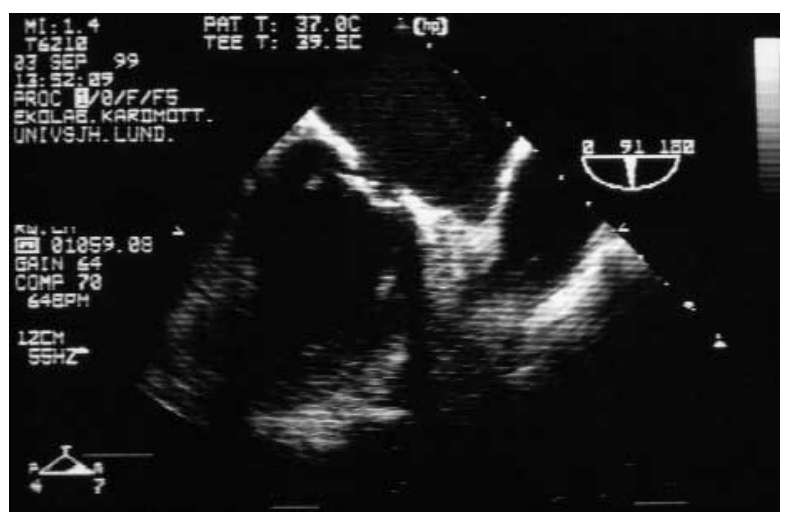

Fig 4. Follow-up echocardiogram (patient 2), showing adequate anterior and PML coaptation.

length of the pericardial patch is dictated by the distance between the midpoint of the posterior mitral anulus and the right fibrous trigone and is usually about $50 \mathrm{~mm}$. The mitral ring annuloplasty was performed on the basis of the measurement of the AML as in the repair of a degenerative mitral valve disease. The appropriate size of the mitral annuloplasty rings together with patch enlargement of the PMLs provided near normal effective mitral orifice areas in relation to the patient's BSA with sufficient relief of MR (Table I). During the follow-up period, there was no increase in the residual MR from one noted at the time of discharge.

With only 2 patients operated on by patch enlargement of the restricted PML and with a follow-up of 34 and 11 months, respectively, we are hesitant to conclude that this technique is applicable in all patients with chronic ischemic MR and restricted PML motion. The surgical strategy should be modified depending on the degree of restriction of the PML motion (partial vs complete) and the extent to which the PML is restricted. A very careful assessment of the ischemic mitral valve pathophysiology by preoperative and intraoperative transesophageal echocardiography is essential for a correct decision-making process. The patch enlargement repair technique in chronic ischemic MR with restricted PML motion offers an anatomic and physiologic correction of the underlying defect and may be considered as an alternative to other presently available surgical techniques.

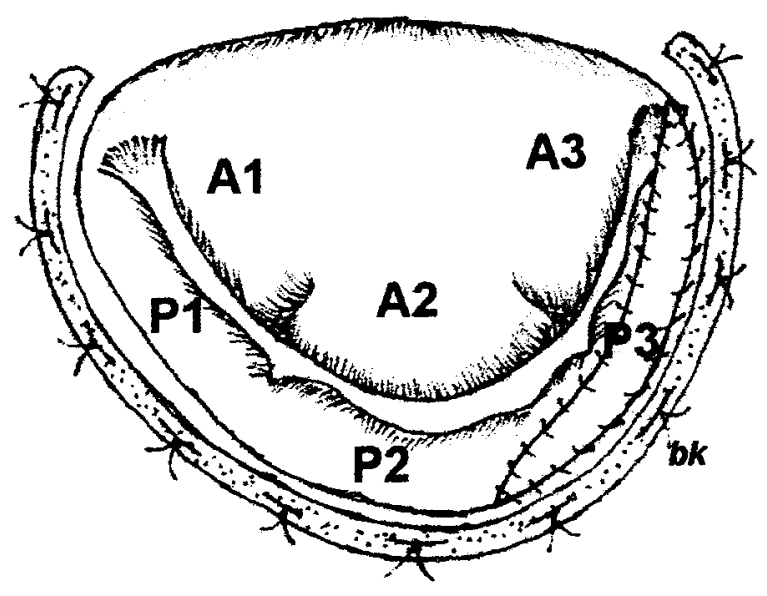

Fig 5. Diagrammatic representation of the mitral valve, showing pericardial patch enlargement of the medial half of $\mathrm{P} 2$, the entirety of $\mathrm{P} 3$, and the posteromedial commissure of the PML and posterior mitral ring annuloplasty. $A 1$ to $A 3$ and $P 1$ to $P 3$ represent anatomic regions of the anterior and posterior mitral valve leaflets, respectively, as described by Carpentier.

\section{REFERENCES}

1. Rankin JS, Hickey MStJ, Smith LR, et al. Ischemic mitral regurgitation. Circulation 1989;79(Suppl):I-116.

2. Dion R, Benetis R, Elias B, et al. Mitral valve procedures in ischemic regurgitation. J Heart Valve Dis 1995;4(Suppl 2):124-31.

3. Hausman H, Siniawski H, Hotz H, et al. Mitral valve reconstruction and mitral valve replacement for ischemic mitral insufficiency. J Card Surg 1997;12:8-14.

4. Gorman JH 3rd, Gorman RC, Jackson BM, et al. Distortions of the mitral valve in acute ischemic mitral regurgitation. Ann Thorac Surg 1997;64:1026-31.

5. He S, Fontaine AA, Schwammenthal E, et al. Integrated mechanism for functional mitral regurgitation. Ann Thorac Surg 1997;64:1026-31.

6. Komeda M, Glasson JR, Bolger AF, et al. Geometric determinants of ischemic mitral regurgitation. Circulation 1997;4:96 (Suppl 9):II-128-33.

\section{Targeted}

The Journal of Thoracic and Cardiovascular Surgery gives you two tables of contents.

The condensed table of contents tells you at a glance what topics and authors are presented each month. The expanded table of contents gives you a brief abstract of each article. You select only those articles of most interest to you for further reading. 